\title{
Computational Geometry for Continuous Resource Location Model in a Competitive Environment
}

\author{
Monika Mangla \\ Research Scholar, TU Patiala \\ Faculty, L.T College of Engineering \\ Navi Mumbai
}

\author{
Deepak Garg \\ Asst. Professor, Computer Science Department \\ Thapar University \\ Patiala
}

\begin{abstract}
Resource location has become a fundamental question to be answered in the day today life. It has found its place in the life for over hundred years from the smallest application like where to locate any electric appliance in a kitchen to opening a new departmental store by a company. Depending upon the context in which the location model is being used, various objective functions may be designed while main features will always remain same [1]. Here in this paper, we have studied the continuous resource location model where competing resources are already known to exist in the sy stem under study. The newly located resources compete for its share of customer demands with the pre-existing resources in the system and with each other also [2]. The paper also discusses how the applications of computational geometry relate to this spatial problem.
\end{abstract}

\section{General Terms}

Resource location, Discrete location model, Network location model, Euclidean distance, $\mathrm{p}$-center location.

\section{INTRODUCTION}

The problem of resource location helps in finding the optimal or near to optimal location for any resource to be located. The location is best determined by some objective function which in turn depends upon the type of resources to be located. Over the time, various researchers have introduced different objective functions and constraints to be looked into. In [1] the complete literature on location problems has been broadly classified into four categories: analytic model, continuous model, network model and discrete location model. In this paper, we are focusing our attention to continuous location model only where resources can be located anywhere on the whole service plane while demand points or clients are located at discrete locations in the plane. The paper aims at finding optimal location of newly placed resources by minimizing some objective function while some resources already exist in the system. The newly placed resources need to compete with the existing resources as well as among themselves to capture a share of market. The paper relates continuous resource location with the applications of computational geometry. The paper is organized as follows.

Here, section II discusses the various types of location models. In this paper, we are limiting our focus to continuous location model only. This section also discusses various constraints that researchers have considered during their research work thus introducing to different types of location model in competitive environment. Following section discusses the applications of computational geometry and how these applications relate to the continuous location models discussed in the previous section.
Finally, Section IV concludes the paper and gives directions for future research.

\section{CONTINUOUS RESOURSE LOCATION}

Continuous location model aims to find location of a resource anywhere in the two dimensional plane while achieving some objective function. This model considers Euclidean distance which may not be possible in some cases thus having limited application. The classical example of continuous location model is Weber problem where a resource is to be located to service $\mathrm{m}$ demand points where each demand point is located at location $\mathrm{x}_{\mathrm{i}}$ and $y_{i}$ for $i$ in $\{1 . . \mathrm{m}\}$ [3]. The location problem can also be extended to multi-facility location also where multiple resources are to be located and existing demand points are to be allocated to resources so that transportation cost is minimized [10].

It should also be considered when to add a new resource into the system as addition of a new resource is a cost affecting decision. The decision parameters for addition of a new resource should be based on the type of resource and initial setup cost. For example whenever a resource is critical like an ambulance which is solved using p-centre problems, whenever radius of the circle becomes more than permissible one, resource needs to be added irrespective of the setup cost as it may result into fatalities [4]. On the other side, if it is regarding an outlet of some departmental store or a restaurant, whenever customer demand exceeds capacity of the existing resources (capacitated resources) by more than adjustable error ratio, it is decided to add a new resource into the system. Error ratio up to some extent may be determined by the initial setup cost of the resource. The size of a resource is determined by the number of resources [5]. For some resources it is preferable to have large number of smaller resources as compared to few large resources as in case of hospitals while on the other hand, resources like amusement parks need to be very large and have high ambience thus increasing the setup cost despite their small number. In [5] attractiveness of a resource for demand points is determined which is based on size of resource, distance of resource and accessibility to resource. To provide a quality of service with some fixed cost, it may also be possible to have a mix of small and large resources in a way so that small facilities are available to all demand points in minimum access time, at the same time these small resources should be capable of providing basic services to the demand points.

In [6] capacitated multi-facility Weber problem is discussed which is concerned with locating $\mathrm{m}$ capacitated resources in the Euclidean plane, and allocation of capacities for $m$ resources among customer demands at a minimum cost. Here, it has been 
assumed that location of customers and demand is known with certainty which may not be possible in every case. Capacity constraint on the resources may result into customers always not patronizing their nearest resources. In this case, unlike full capture model [7][8] the customer demand may be diverted to different resources. The cases, where demand point locations are not known with certainty result into probabilistic capacitated multi-facility Weber problem where customer locations is two dimensional random vector distributed according to some probability density function [6]. Rigorous research has been carried out by various researchers to solve this capacitated multi-facility Weber problem using the probability density function representing the location of various customer locations or demand points.

There is a thorough research in the area of location of resources when the demand of clients is uncertain. Various researchers have proposed different techniques for handling the issue. In [9] author has proposed to locate the resource at a position that minimizes the regret if location was not optimal, that will be providing service to $\mathrm{p}$ demand points out of total $\mathrm{n}$ demand points existing in the system. Apart from various issues related to location models and cost, in [11] author has addressed an investment issues regarding whether to invest in an existing resource or add a new resource to the system. Author has proposed that capacity of resource can be stretched up to some extent by incurring some additional cost. While researchers have addressed various issues while locating resources on a two dimensional plane, in [12] author has discussed multi-objective solution for uncapacitated resources. Researchers have also discussed various parameters while allocating demand points to the resources. For uncapacitated resources, customer demands were always allocated to the nearest resource, which may not be possible in capacitated resources always. In [13] author has proposed various heuristics for multiple resource location of a franchise in a competitive environment with an objective to capture whole market share by the franchise.

Various proposals by different researchers have their own considerations to find location of single or multiple resources. Hakimi and Drezner have assumed that a customer always patronizes its closest resource. Later Huff introduced a realistic approach by suggesting that customer divide their demand according to some gravity-based formula, which suggests that probability of a customer patronizing a facility is proportional to its attractiveness and inversely proportional to the distance from customer [13].

All these variations in the continuous location model have their own implications and are solved by researcher using some or other tools. Some researchers have solved the location problem by analytic methods for minimizing objective function. Some researchers have tried their hand on various heuristics to find optimal location for the resource in a competitive environment. Intelligence based methods have also been a tool of choice in various researchers' work. Computational geometry has also proved itself in the area of solving various spatial problems. Computational geometry has been a vital choice for solving various spatial problems by the researchers over the last decade.

\section{COMPUTATIONAL GEOMETRY}

Computational geometry has been accepted as an efficient area of engineering for developing algorithm and data structures in terms of geometric objects. Some of the problems in computational geometry come from applications in pattern recognition, computer graphics, statistics and robotics etc., thus there is hardly any area left, where computation geometry has not proved its capability as an efficient tool [14][15]. This branch of en gineering is capable of solving spatial problems as well in an efficient and effective manner. Computational geometry is well versed in solving static applications like range searching, point location etc. and dynamic problems like motion planning and routing etc. The various applications of computational geometry are following.

\subsection{Voronoi Diagram}

It partitions the space into regions where all points in the region are closer to the region's data point than to any other data point.

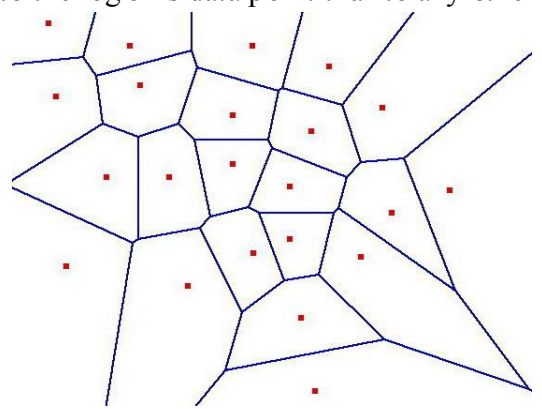

Fig 1: Voronoi Diagram for sites

Various algorithms are existing in literature for its computation [14][15]. Typically the set $P$ of points is viewed as being static, although in many applications it is natural to consider point sets (sites) that changes over time. This dynamic nature requires reconstruction of $\operatorname{VOD}(\mathrm{P})$ thus leading to significant increase in the computational time complexity. In [16] G.R.,Samet et al. [6] have proposed a $\mathrm{O}(\mathrm{n})$ time algorithm to update VOD by using a simple data structure called as Voronoi tree or V-tree in short $[17,18]$. It requires $\mathrm{O}(\mathrm{n} \log \operatorname{logn})$ storage, but this requirement can be reduced by taking advantage of a storage/deletion time tradeoff.

\subsection{Delaunay Triangulation}

Delaunay triangulation is the dual of voronoi diagram. This graph $\mathrm{G}$ has a node for every Voronoi cell equivalently, and for

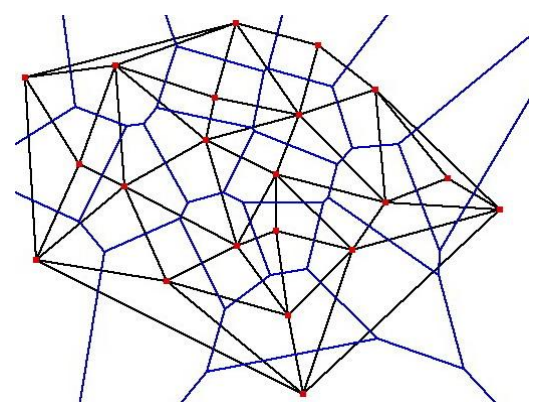

Fig 2: DelaunayTriangulation for Fig 1.

every site it has an arc between two nodes if the corresponding cells share an edge. Note that this means that $G$ has an arc for every edge of Vor(P). As you can see in Fig 2(the dual of Fig 1) there is a one-to-one correspondence between the bounded faces of $\mathrm{G}$ and the vertices of $\operatorname{Vor}(\mathrm{P})$. 


\subsection{Convex Hull}

Convex hull for points $\mathrm{P}$ is the minimum enclosing poly gon that encloses all points in set $\mathrm{P}$. It is also called minimum bounding region that bounds all points in set $\mathrm{P}$. Various algorithms have been proposed by researchers to compute convex hull of set $\mathrm{P}$. As far as location models are concerned, if set $\mathrm{P}$ represents set of all demand points then all resources should surely lie in the convex hull of set $\mathrm{P}$. No resource should be placed outside the set $\mathrm{P}$, which reduces the cost of optimal location computation up to significant level.

\section{COMPUTATIONAL GEOMETR Y IN CONTINUOUS LOCATION MODEL}

Computational geometry is capable of solving spatial problems in an effective and efficient manner. Here, in this paper we have shown how computational geometry applications can be used for continuous resource location model in a competitive environment i.e where competing agencies already exist in the market.

Voronoi diagram can be used for locating resources of a follower company B when leader company A already exists in the market. Let $\mathrm{n}$ outlets of Leader Company A exists in the market each positioned at location $a_{i}$ for $1 \leq \mathrm{i} \leq \mathrm{n}$ and capacity of each resource of company $\mathrm{A}$ is represented by $\mathrm{CA}_{\mathrm{i}}$ again for $1 \leq$ $\mathrm{i} \leq \mathrm{n}$. The location of Leader Company is shown in the voronoi diagram with red color. Now Follower Company wants to locate its resources, using voronoi diagram it is observed that follower company B should minimally locate its outlets at locations shown by symbol @, which is intersection of three voronoi cells representing three outlets of company $A$ i.e the outlet $b_{i \mathrm{ij}}$ will

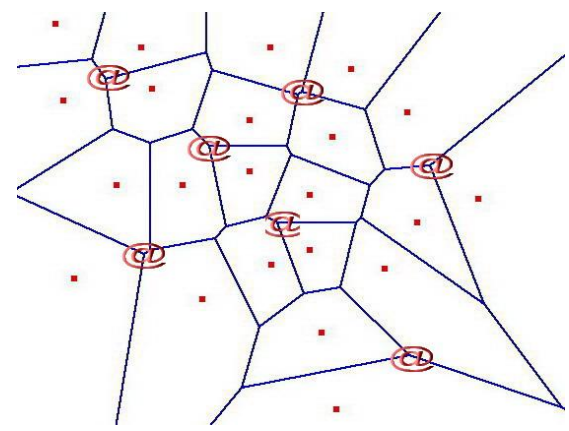

Fig 3: position marked with @ represents location of Follower Company Resources

be located at intersection of voronoi cell of resource $a_{i}, a_{j}$ and $a_{k}$ as shown in Fig. 3. Initially, the capacity of resource $b_{i j k}$ represented by $\mathrm{CB}_{\mathrm{ijk}}$ should be maximum of $\mathrm{CA}_{\mathrm{i}}, \mathrm{CA}_{\mathrm{j}}$ and $\mathrm{CA}_{\mathrm{k}}$.

$$
\mathrm{CB}_{\mathrm{ijk}}=\max \left(\mathrm{CA}_{\mathrm{i}}, \mathrm{CA}_{\mathrm{j}}, \mathrm{CA}_{\mathrm{k}}\right)
$$

Later the decision can be taken whether capacity of existing resources should be stretched or new resources should be added. The locations $b_{i j k}$ ensure to achieve minimum average distance from all demand points in the regions allocated to $a_{i}, a_{j}$ and $a_{k}$ thus implementing median problem. To further increase the attractiveness of outlet of follower company's, some policies may be designed that may lure maximum market share by the company against all competing agencies.
Delaunay triangulation, the dual of voronoi diagram can also be used to handle the situation when a resource of a company needs to be removed due to any reason. Whenever a resource is to be removed, the voronoi diagram can be constructed with n-1 resources as shown in the following diagram. Now suppose in the Fig. 4 the resource marked with the symbol \# is to be removed. The resources marked with symbol $\$$ represent the resources which are located at a distance of path length 1 from the same resource in the Delaunay triangulation. It can be implemented by drawing the voronoi diagram with $n-1$ resources

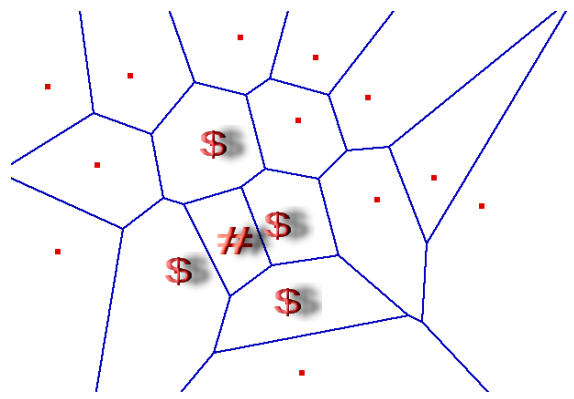

Fig 4: Symbol \# represents the resource to be removed

as shown in the following Fig 5. The customers that were initially allocated to the resource marked with symbol \# will be distributed among the resources marked with $\$$ sy mbol.

Now allocation of demand points can be easily handled with the help of voronoi cells of $n-1$ points. Whenever there is some capacity constraint with assigned resource, demands may be allocated to the resources which are allocated at a length of one

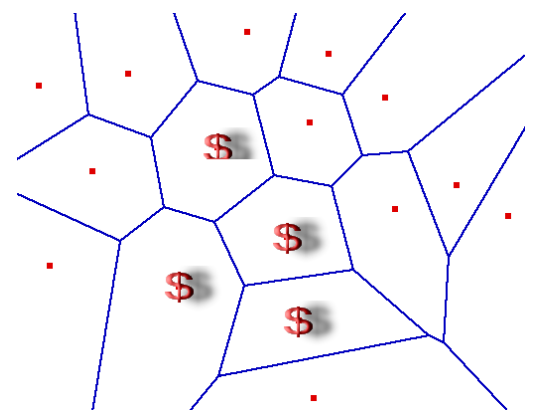

Fig 5: Voronoi Diagram after resource removed

from the assigned resource and have capacity to accommodate further demands made by customers. All customers lying on the boundary of a voronoi cell may be allocated to the resource having maximum difference of capacity and currently allocated customers.

The decision of opening a new outlet or stretching the capacity of existing resource can also be handled. It may be decided by observing the additional cost for stretching the capacity of an existing resource and opening a new resource. If the additional cost for updating an existing resource is let us say up to $\alpha$ percent of that for opening a new store, it is preferable to invest into an existing resource. The value of $\alpha$ needs to be decided with thorough investigation and depends upon type of resource also. Thus, there are many other applications which can be 
handled by various applications available in computational geometry.

\section{CONCLUSION}

Here, in this paper continuous location of resources in a competitive environment have been discussed. Then applications of computational geometry are discussed and how these applications can be used for finding the optimal locations in a competitive environment. The research work can be further extended in the direction of policy for reaction of Follower Company. It can also be extended to incorporate when multiple competing agencies exist in the market as in this paper only one competitor has been discussed.

\section{REFERENCES}

[1] C.S. ReVelle, H.A. Eiselt, M.S. Daskin, "A bibliography for some fundamental problem categories in discrete location science", Proc. European Journal of Operational Research184, pp 817-848, 2008.

[2] Robert Aboolian, Oded Berman, Dmitry Krass, "Competitive facility location and design problem", Proc. European Journal of Operational Research182, pp 40-62, 2007.

[3] Zvi Drezner,Horst W. Hamacher, "Facility Location: Applications and Theory", Springer- verlag, first edition 2001, ISBN 3-540-21345-7.

[4] Mark S. Daskin. Network and Discrete Location Models, Algorithms, and Applications, wiley- intersciences, ISBN 0-471-01897-X

[5] Zhishuang yao, Loo hay lee, Wikrom jaruphongsa, Vicky Tan, Chen Fei Hui, "Multi-source facality locationallocation and inventory problem", Proc. European Journal of Operational Research 207, pp 750-762, 2010.

[6] Kuban Altınel, Engin Durmaz, Necati Aras, Kerem Can Ozkısack, "A location-allocation heuristic for the capacitated multi-facility Weber problem with probabilistic customer locations", Proc. European Journal of Operational Research 198, pp 790-799, 2009.

[7] H.A. Eiselt, G. Laporte, Demand allocation functions, Location Science 6 (1998) 175-187
[8] S.L. Hakimi, Locations with spatial interactions: Competitive locations and games, in: P.B. Mirchandani, R.L. Francis (Eds.),Discrete Location Theory, Wiley Interscience, 1990.

[9] Eduardo Conde, "Minmax regret location-allocation problem on a network under uncertainty", Proc. European Journal of Operational Research 179, pp 1025-1039,2007

[10] Martin Bischoff, Kerstin Dachert, “Allocation search methods for a generalized class of location-allocation problems", Proc. European Journal of Operational Research 192, pp 793-807, 2009

[11] Joseph Harkness, Charles ReVelle, "Facility location with increasing production costs", Proc. European Journal of Operational Research145, pp 1-13, 2003.

[12] Elena Fernandez, Justo Puerto, "Multiobjective solution of the uncapacitated plant location problem", Proc. European Journal of Operational Research145, pp 509-529, 2003.

[13] Tammy Drezner, Zvi Drezner, Said Salhi, "Solving the multiple competitive facilities location problem", Proc. European Journal of Operational Research 142, pp 138151,2002

[14] Mark de Berg, Otfried Cheong, Marc van Kreveld, Mark overmars, "Computational Geometry- Algorithms and Applications",Springer-Verlag, Third edition, 2008

[15] Joseph O' Rourke, "Computational Geometry in C", Cambride University, second edition, 1997

[16] G. R. Hjaltason and H. Samet. Properties of embedding methods for similarity searching in metric spaces. IEEE Transactions on Pattern Analysis and Machine Intelligence, 25(5):530-549, May 2003. Also University of Maryland Computer Science TR-4102.

[17] F. K. H. A. Dehne and H. Noltemeier. "Voronoi trees and clustering problems". Proc. IEEE Int'l Conf., Inf. Syst., vol. 12 No.2, pp.171-175, 1987.

[18] G. Albers, L.J Guibas, J.S.B.Mitchell, and T. Roos, "Voronoi diagrams of moving points", Presented at Intl. Journal on Computational Geometry and Applications, vol. 8, no. 3, pp.365-380, 1998. 